\title{
O Envelhecimento Pulmonar e as suas Alterações Imagiológicas
}

\section{Lung Aging and its Imagiological Changes}

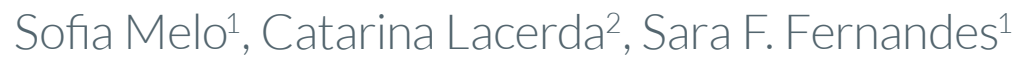

Autor Correspondente/Corresponding Author: Sofia Melo [asofiabmelo@gmail.com]

\section{RESUMO}

INTRODUÇÃO: Com o aumento da esperança média de vida, as populações atingem idades cada vez mais tardias. Como consequência, é essencial que se faça uma distinção clara das alterações que se consideram fisiológicas do envelhecimento, das patológicas.

O objetivo desta revisão foi identificar as principais alterações imagiológicas do parênquima pulmonar que surgem com o envelhecimento, presentes na radiografia torácica e tomografia computorizada.

MATERIAL E MÉTODOS: Os critérios de inclusão definidos foram artigos que descrevessem achados imagiológicos em radiografia torácica e tomografia computorizada atribuídos ao envelhecimento em idosos com idade igual ou superior a 65 anos. Os artigos exclusivamente referentes a alterações da função pulmonar, parede torácica e mediastino foram excluídos, assim como, artigos duplicados e redigidos noutras línguas. Além disso, a população não podia apresentar história de tabagismo atual ou passado; exposições ocupacionais relevantes, por exemplo, trabalham na indústria de amianto; espirometria a documentar obstrução; dispneia documentada por mMRC > 2 ou outros marcadores de comprometimento pulmonar.

RESULTADOS: Há um aumento da hiperinsuflação pulmonar de forma homogénea e de fenómenos de air-trapping. As opacidades de vidro despolido e o padrão de mosaico do parênquima pulmonar são também frequentes nestas populações, contudo não se pode excluir a possibilidade de patologia pulmonar subjacente. O diagnóstico de bronquiectasias requer critérios próprios nos idosos e os nódulos pulmonares devem ser abordados de acordo com as normas publicadas.

DISCUSSÃO/CONCLUSÃO: Com o envelhecimento estão objetivadas alterações do parênquima pulmonar, contudo a distinção entre o patológico e o fisiológico é ainda difícil de definir. 
PALAVRAS-CHAVE: Envelhecimento/fisiologia; Pulmão/diagnóstico por imagem; Radiografia Torácica; Tomografia Computorizada

\section{ABSTRACT}

INTRODUCTION: With the increase in the average of life expectancy, populations reach increasingly late ages. Therefore, it is essencial to make a clear distinction from changes that are considered to be physiological from aging, from pathological ones.

The objective of this review was to identify the main imaging abnormalities of the pulmonary parenchyma that appear with aging, present in the thoracic radiography and computed tomography.

MATERIAL AND METHODS: The inclusion criteria were articles describing imaging findings in thoracic radiography and computed tomography attributed to aging in the elderly aged 65 years or older. The articles exclusively related to changes in lung function, chest wall and mediastinum were excluded, as well as duplicated articles and written in other languages. In addition, the population could not have current or past smoking history; relevant occupational exposures, eg. work in the asbestos industry; airway obstruction on the spirometry; dyspnea documented by $m M R C>2$ or other markers of lung involvement.

RESULTS: There is an increase in a homogeneous pulmonary hyperinflation and air-trapping. Ground glass opacities and the mosaic pattern of the pulmonary parenchyma are also frequent in these populations, but the possibility of underlying pulmonary pathology cannot be ruled out. Diagnosis of bronchiectasis requires proper criteria in the elderly and the pulmonary nodules should be managed according to published guidelines.

DISCUSSION/CONCLUSION: With aging changes in the pulmonary parenchyma are objectified, but the distinction between the pathological and the physiological is still difficult to define.

KEYWORDS: Aging/physiology; Lung/diagnostic imaging; Radiography, Thoracic; Tomography, X-Ray Computed

\section{INTRODUÇÃO}

Atualmente, a par do envelhecimento progressivo da população e ao crescente aumento da esperança média de vida, deparamo-nos frequentemente com exames imagiológicos do parênquima pulmonar realizados em idades mais tardias.

Devido a isto, é essencial que se faça uma distinção clara das alterações que se consideram fisiológicas do enveIhecimento, das patológicas. E este é o desafio, pois nos idosos, é difícil estabelecer o que é a normalidade, devido às numerosas modificações anatómicas e fisiológicas que ocorrem durante o envelhecimento. ${ }^{1}$

Ao longo da vida ocorrem alterações a nível estrutural e funcional das vias aéreas, do parênquima pulmonar e dos músculos respiratórios² com diminuição do fluxo aéreo. Além disso, surgem alterações na coluna vertebral, costelas, articulações e músculos que afetam negativamente a normal função pulmonar. ${ }^{3}$ Verifica-se uma diminuição da capacidade de eliminar secreções pulmonares, quer pelo enfraquecimento da clearance mucociliar, quer devido à redução da força da tosse. ${ }^{4}$ Há ainda um declínio imunológico aumentando a suscetibilidade a infeções respiratórias. ${ }^{3}$ A nível histopatológico verifica-se uma deposição excessiva de proteínas de colágeno inversamente às fibras de elastina, resultando numa remodelação da matriz extracelular do parênquima pulmonar. ${ }^{3}$

Por isto, esta revisão, tem como objetivo identificar as principais alterações imagiológicas do parênquima pulmonar que surgem com o envelhecimento, presentes na radiografia torácica (RT) e tomografia computorizada (TC).

\section{MATERIAL E MÉTODOS}

Realizou-se um estudo retrospetivo e descritivo, dos artigos publicados entre janeiro de 2014 e setembro de 2018, publicados na base de dados PubMed ${ }^{\circledR}$, Cochrane Library $^{\circledR}$ e DARE ${ }^{\circledR}$, com os termos MeSH "Aging"; "Lung"; "Diagnostic imaging", redigidos na língua inglesa e portuguesa.

Os critérios de inclusão definidos foram artigos que descrevessem achados imagiológicos em RT e TC atribuídos ao envelhecimento em idosos com idade igual ou superior a 65 anos.

Os artigos exclusivamente referentes a alterações da função pulmonar, parede torácica e mediastino foram excluídos, assim como, artigos duplicados e redigidos noutras línguas. Além disso, a população incluída não podia apresentar história de tabagismo atual ou passa- 
do; exposições ocupacionais relevantes, por exemplo, trabalham na indústria de amianto; espirometria a documentar obstrução; dispneia documentada por mMRC >2 ou outros marcadores de comprometimento pulmonar.

\section{RESULTADOS}

Da pesquisa realizada foram encontrados 90 artigos, não havendo artigos duplicados. Após leitura de título e resumo selecionaram-se sete para leitura integral. Após isto, foram incluídos cinco artigos, tendo-se excluídos dois por não se enquadrarem no âmbito do tema e população definida (Fig. 1). Para estratificar o nível de evidência e a força de recomendação utilizou-se a escala Strength of Recommendation Taxonomy (SORT) da American Family Physician. ${ }^{5} \mathrm{Na}$ Tabela 1 estão de forma sucinta explicitadas as principais conclusões dos artigos selecionados, assim como a sua tipologia.

O conhecimento do processo de envelhecimento pulmonar e das suas traduções imagiológicas é fundamental de forma a distinguirmos o fisiológico do patológico e, assim, orientarmos de forma mais correta o doente. De seguida, estão documentadas as principais alterações do envelhecimento do parênquima pulmonar a nível imagiológico que foram descritas nos artigos selecionados, assim como uma breve definição destas.

\section{AIR-TRAPPING E HIPERINSUFLAÇÃO PULMONAR}

Uma das primeiras alterações fisiopatológicas que se observa com o envelhecimento é a perda de elasticidade pulmonar, agravada pelo aumento da rigidez da caixa torácica e pela redução da força dos músculos respiratórios. ${ }^{10}$ Isto resulta numa dilatação do espaço aéreo, maior colapsibilidade das pequenas vias aéreas e uma

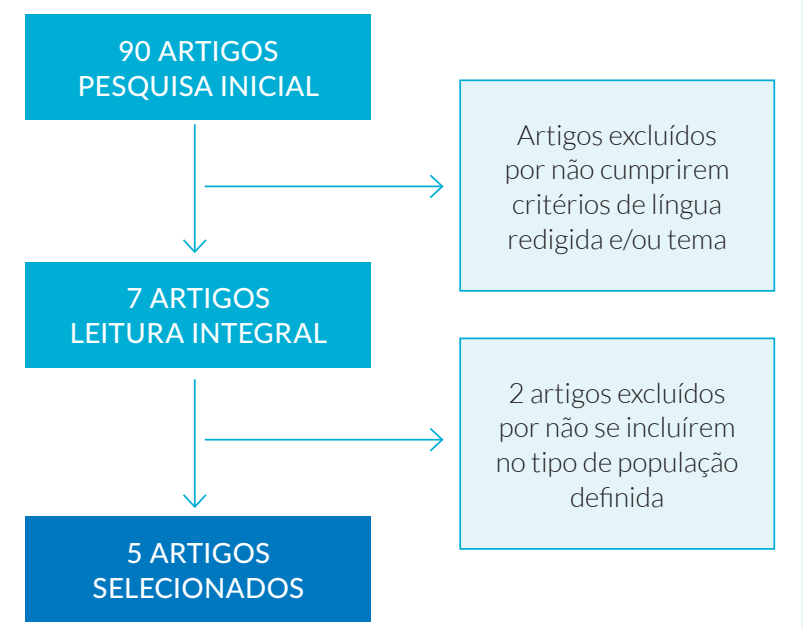

FIGURA 1. Fluxograma de seleção dos artigos.
TABELA 1. Artigos selecionados e suas conclusões.

Referência Tipo de Artigo

Schröder TH, et al (2015)

Revisão

2

- Aumento do tamanho fisiológico dos alvéolos e dos ductos alveolares contrasta com a redução da densidade;

- Hiperinsuflação alveolar distribuída homogeneamente por todo o pulmão, enquanto que as alterações pulmonares enfisematosas afetam principalmente o lobo superior ou inferior;

- Opacidade de vidro despolido relativamente homogénea e reversível nas regiões basais dependentes do pulmão, possivelmente devido à inspiração superficial e alterações dependentes da posição (hipostática) do parênquima;

- Padrão de mosaico frequentemente limitado ao lóbulo secundário ou air-trapping durante a expiração, com uma diminuição da densidade do parênquima pulmonar;

- Nódulos pulmonares: a sua vigilância deve seguir as normas publicadas, não havendo diferenças nos idosos;

- Bronquiectasias: devem ser usados critérios adicionais para o seu diagnóstico: incluir uma relação broncoarterial maior que 1,5.

\begin{tabular}{|l|l|l} 
Martinez $\mathrm{CH}$, et al $(2017)^{7}$ & Transversal
\end{tabular}

- Perda do recolhimento elástico;

- Aprisionamento de ar;

- Diminuição da capacidade vital e aumento do volume residual;

- Hiperinsuflação homogénea;

- Aumento das anormalidades das pequenas vias aéreas cerca de 3,1\% por década de idade (intervalo de confiança de 95\% [IC], $2,6 \%-3,5 \%)$.

Winter D H, et al (2015) ${ }^{8}$ Estudo Caso-Controlo 2

- Diminuição da densidade do parênquima pulmonar;

- Redução da sua complexidade microestrutural;

- Air-trapping;

- Dilatação de pequenas vias aéreas;

- Aumento de bandas parenquimatosas, opacidades em vidro despolido e linhas septais, mais no pulmão direito, micronódulos, opacidades reticulares, cistos pulmonares e bronquiectasias;

- Faixas parenquimatosas, linhas septais e opacidades em vidro despolido foram marcadamente predominantes nas zonas pulmonares inferiores.

Occhipinti M, et al $(2017)^{2} \quad$ Estudo de Coorte 2

- Dilatação brônquica;

- Valor médio de razão bronco-arterial significativamente maior;

- Espessamento da parede brônquica;

- Air-trapping cuja extensão tem correlação significativa com a idade, bem como com história de tabagismo.

\begin{tabular}{l|l|l} 
Copley S J, et al $(2016)^{9}$ & Estudo Caso-Controlo
\end{tabular}

- Hiperinsuflação pulmonar;

- Dilatação alveolar;

- Gânglios linfáticos perifissurais são achados comuns, mais frequentes no sexo masculino, com distribuição subpleural e pulmonar inferior, não calcificados, diâmetro <10 mm, morfologia oval, lentiforme ou triangular e distribuição perilinfática;

- Air-trapping aumenta significativamente com a idade;

- Correlação significativa entre idade e aumento da razão broncoarterial;

- Dilatação brônquica e espessamento parietal foi significativamente mais frequente e extensa com predominância do lobo inferior;

- Bronquiectasias detetadas por TC mais frequentes no grupo mais idoso em comparação com o grupo mais jovem com diminuição da depuração mucociliar e reflexo da tosse;

- Padrão reticular focal associado à osteofitose torácica;

- Cistos mais prevalentes, 7,6\% a 25\%, no grupo de idosos, distribuídos uniformemente, sem correlação com tabagismo;

- Bandas parenquimatosas, opacidades em vidro despolido, linhas septais, micronódulos e opacidades reticulares também foram mais prevalentes. 
redução nos volumes expirados, ${ }^{10}$ logo um aumento do volume residual. Esta é a base fisiopatológica do air-trapping e da hiperinsuflação pulmonar bastante características na população idosa.

Assim, o air-trapping, definido com um aprisionamento de ar nas vias aéreas distais, na TC apresenta-se como uma diminuição da atenuação do parênquima pulmonar, especialmente durante a expiração, permanecendo mais lucente do que o tecido pulmonar circundante. ${ }^{11}$ Todos os artigos são uníssonos quanto ao seu aparecimento, sendo que Occhipinti $M$ et $a^{2}$ refere uma correlação significativa com a idade descrevendo uma frequência de $76 \%$ na população idosa. Paralelamente, afirma que há uma associação positiva entre o envelhecimento e o atingimento das pequenas vias aéreas, aumentando por década 3,6\%, ${ }^{2}$ já Martinez $\mathrm{CH}$ et al refere uma taxa de cerca de $3,1 \%$. $^{7}$

A nível imagiológico, a hiperinsuflação pulmonar associada ao envelhecimento, segundo Schröder TH et al e Martinez C H et al, é distribuída de forma homogénea pelo parênquima pulmonar, ao contrário da hiperinsuflação observada no enfisema que se localiza principalmente no lobo superior ou inferior, de acordo com a etiologia (Fig. 2). ${ }^{12}$ Além disso, imagiologicamente a

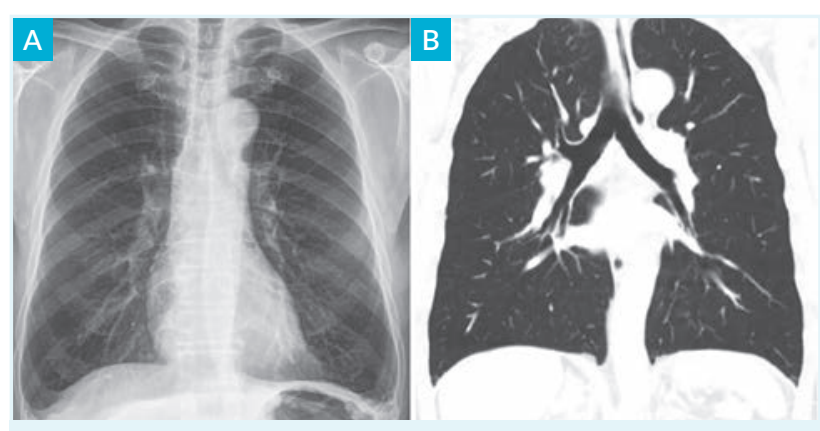

FIGURA 2. Hiperinsuflação pulmonar relacionada com a idade num homem de 69 anos não fumador. A: Radiografia torácica. B: Tomografia computorizada. Adaptado de Kang EY, et al. J Korean Soc Radiol 2017;77:166-76. ${ }^{20}$

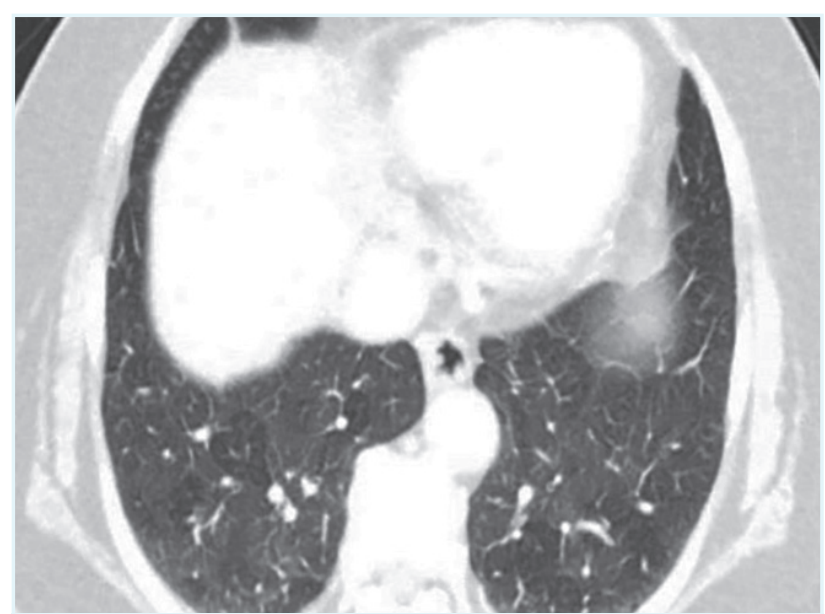

FIGURA 3. Padrão de mosaico numa mulher de 76 anos. Adaptado de Kang EY, et al. J Korean Soc Radiol 2017;77:166-76.20 hiperinsuflação que ocorre nos idosos não apresenta sinais inflamatórios associados e a capacidade pulmonar total permanece inalterada. ${ }^{1}$

\section{OPACIDADE EM VIDRO DESPOLIDO}

A opacidade em vidro despolido apresenta-se como uma opacidade difusa do pulmão, com preservação das margens brônquica e vascular, contrastando com a consolidação, que é definida como um aumento homogéneo da atenuação do parênquima que obscurece as margens dos vasos e paredes das vias aéreas. ${ }^{13}$ Estas opacidades na TC são secundárias à redução de ar e preenchimento parcial dos espaços aéreos alveolares, espessamento do interstício e das paredes alveolares, aumento relativo da perfusão ou a uma combinação destes. ${ }^{13}$

Segundo um dos artigos, estas opacidades são relativamente homogéneas e reversíveis nas regiões basais do pulmão, possivelmente devido à inspiração superficial (hipoventilação) e alterações dependentes da posição do idoso. ${ }^{12}$

\section{PADRÃO DE MOSAICO}

$\mathrm{Na}$ TC, o padrão de mosaico é representado como uma atenuação pulmonar variável que resulta numa aparência heterogénea do parênquima (Fig. 3). ${ }^{14}$ Uma das suas principais dificuldades é diferenciar quais as áreas que são normais, das anormais. ${ }^{14}$ Para isso, um dos melhores métodos de distinção é realizar uma imagem em expiração. Nos idosos, está frequentemente limitado ao lóbulo secundário ou devido a air-trapping durante a expiração, com uma consequente diminuição da densidade do parênquima. ${ }^{12}$ Porém, de salientar que estas alterações também podem ser sinal de doença pulmonar.

\section{NÓDULOS PULMONARES}

Os nódulos pulmonares são estruturas arredondadas, bem ou mal circunscritos, que na imagem surgem como opacidades focais, que pela definição tradicional têm $\leq 3 \mathrm{~cm}$ de diâmetro e são circundados por pulmão arejado. ${ }^{14}$ Podem ser únicos ou múltiplos e não apresentam anormalidades associadas no tórax, como linfadenopatia ou doença pleural. ${ }^{14}$ Nos idosos a sua vigilância e abordagem devem ser de acordo com as normas publicadas. $^{12}$

Segundo Copley SJ et al os gânglios intrapulmonares benignos/gânglios linfáticos perifissurais, embora se tenha dados limitados, são achados comuns, nos indivíduos de 50 a 75 anos, que participam nos programas de rastreio de neoplasia pulmonar. ${ }^{9}$ São mais frequentes no sexo masculino, com uma distribuição subpleural e pulmonar inferior, não calcificados, diâmetro $<10$ mm, morfologia oval, lentiforme ou triangular e distribuição perilinfática. ${ }^{9}$ 


\section{BRONQUIECTASIAS}

As bronquiectasias (Fig. 4) são caracterizadas por um alargamento irreversível das vias aéreas de médio a pequeno calibre, com destruição das paredes brônquicas, associadas a inflamação, infeção bacteriana crónica. ${ }^{15}$ Geralmente, surgem após uma infeção respiratória e originam tosse crónica, produção de expetoração abundante, por vezes purulenta, e infeções recorrentes. ${ }^{15}$ As bronquiectasias, não devidas à fibrose cística, são evidenciadas imagiologicamente pela dilatação permanente dos brônquios. ${ }^{16}$ De acordo com o estudo realizado por Matsuoka et al há uma relação significativa entre o envelhecimento e a razão entre o diâmetro do brônquio e a artéria acompanhante - razão broncoarterial - sendo esta influenciada pela idade. ${ }^{17}$ Porém, a hipóxia não é um evento fisiologicamente relacionado à idade. ${ }^{2}$ Assim, o diagnóstico de bronquiectasias na população idosa deverá ser feito com precaução.

Em 2015, no estudo realizado por Winter et al, que compara os achados imagiológicos de 47 idosos versus 24 jovens, refere uma taxa de bronquiectasias de $14,9 \%{ }^{8}$

\section{OUTRAS CARACTERÍSTICAS PARENQUIMATOSAS}

Além das características parenquimatosas anteriormente referidas, de acordo com Copley SJ et al e Schröder TH et al, no envelhecimento ocorre ainda um padrão reticular focal associado à osteofitose torácica, com uma taxa de 45\%; espessamento reticular subpleural e basal (espessamento do septo em 60\% do grupo de pessoas com mais de 75 anos); cistos distribuídos uniformemente, em 7,6\% a 25\%.9,12 Juntamente a estes achados, também ocorre um aparecimento de bandas parenquimatosas, micronódulos, opacidades reticulares e linhas

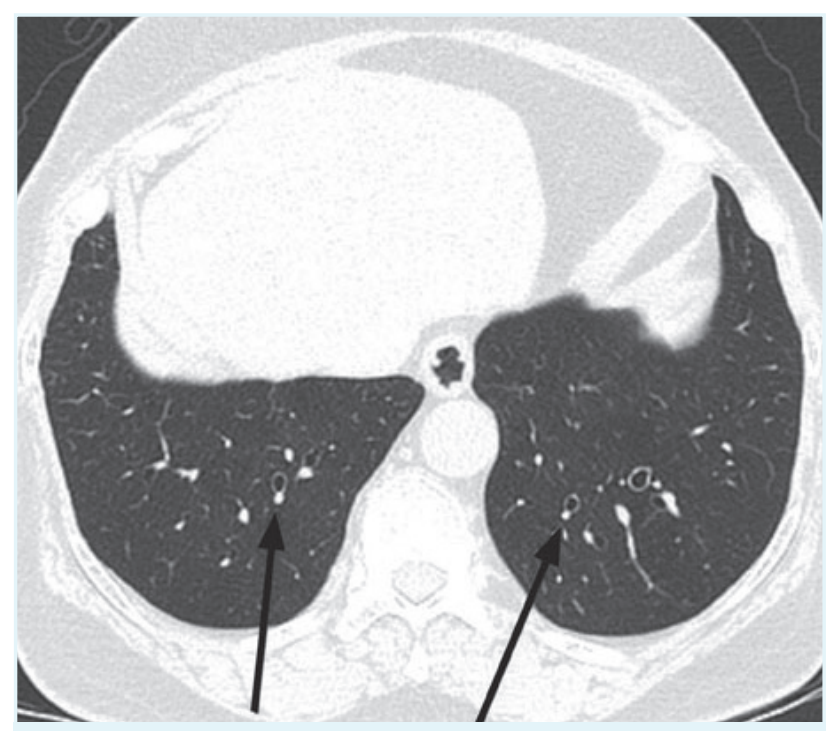

FIGURA 4. Aumento da razão broncoarterial numa mulher de 76 anos assintomática e sem doença pulmonar conhecida. Adaptado de Kang EY, et al. J Korean Soc Radiol 2017;77:166-76. ${ }^{20}$ septais, estas mais frequentes nas zonas pulmonares inferiores. ${ }^{8}$

\section{DISCUSSÃO/CONCLUSÃO}

Uma das principais dificuldades em estudos do envelhecimento é a sua própria definição, podendo ser descrito como "o declínio progressivo da homeostase que ocorre após a fase reprodutiva da vida estar completa, resultando num aumento do risco de doença ou morte". ${ }^{18}$

Após análise dos artigos incluídos nesta revisão verifica-se que com o envelhecimento ocorre uma predominância da hiperinsuflação pulmonar homogénea nesta população, assim como, fenómenos de air-trapping. A presença de opacidades de vidro despolido e padrão de mosaico do parênquima pulmonar, bandas parenquimatosas, linhas septais, opacidades reticulares e cistos pulmonares são também mais prevalentes. ${ }^{19,20}$ Assim, a sua presença não deverá ser considerada, à partida, patológica e após exclusão de possível doença encarado como algo fisiológico. Quanto às bronquiectasias o seu diagnóstico terá de ser acautelado e os nódulos pulmonares devem ser abordados de acordo com as normas publicadas.

Os estudos existentes são parcos e com amostras bastante reduzidas, para além que devido à exposição a poluentes ambientais a definição de envelhecimento de forma natural possa sofrer um viés. Além disso, são poucos deles que recorrem a exames imagiológicos para a sua melhor caracterização, havendo apenas estudos com base em revisão literária.

Apesar das várias tentativas de definir de forma clara e objetiva quais as características do parênquima pulmonar que podem ser atribuídas ao seu envelhecimento, a margem de certeza é dúbia, sendo por isso, essencial a comparação com exames prévios. Por vezes, o acompanhamento das lesões em detrimento de uma intervenção deve ser ponderado, cumprindo o princípio hipocrático do primum non nocere.

Concluindo, perante uma população progressivamente mais envelhecida, existem alterações a nível imagiológico do parênquima pulmonar que devem ser objetivadas. Contudo, a classificação definitiva de características consideradas "normais" para a idade é difícil sendo necessários estudos com amostras mais amplas.

CONFLITOS DE INTERESSE: Os autores declaram não ter qualquer conflito de interesse na realização do presente trabalho.

FONTES DE FINANCIAMENTO: Não houve qualquer fonte de financiamento na realização do presente trabalho. 
PROVENIÊNCIA E REVISÃO POR PARES: Não comissionado; revisão externa por pares.

CONFLICTS OF INTEREST: The authors declare that they have no conflicts of interest.

FINANCIAL SUPPORT: This work has not received any contribution, grant or scholarship.

PROVENANCE AND PEER REVIEW: Not commissioned; externally peer reviewed.

\section{REFERÊNCIAS}

1. Bonomo L, Larici AR, Maggi F, Schiavon F, Berletti R. Aging and the respiratory system. Radiol Clin North Am. 2008;46:685702, v-vi. doi: 10.1016/j.rcl.2008.04.012.

2. Occhipinti M, Larici AR, Bonomo L, Incalzi RA. Aging airways: between normal and disease. A multidimensional diagnostic approach by combining clinical, functional, and imaging data. Aging Dis. 2017;8:471-85. doi: 10.14336/AD.2016.1215.

3. Ciccarese F, Chiesa M, Vizioli L, Pasquali M, Zoli M. The senile lung as a possible source of pitfalls on chest ultrasonography and computed tomography. Respiration. 2015:90:56-62. doi: 10.1159/000430994.

4. Lowery EM, Brubaker AL, Kuhlmann E, Kovacs EJ. The aging lung. Clin Interv Aging. 2013;8:1489-96. doi: 10.2147/CIA. S51152.

5. Ebell MH, Siwek J, Weiss BD, Woolf SH, Susman J, Ewigman $\mathrm{B}$, et al. Strength of Recommendation Taxonomy (SORT): a patient-centered approach to grading evidence in the medical literature. Am Fam Physician. 2004;69:548-56.

6. Schroder TH, Storbeck B, Rabe KF, Weber C. The aging lung: clinical and imaging findings and the fringe of physiological state. Rofo. 2015;187:430-9. doi: 10.1055/s-0034-1399227.

7. Martinez CH, Diaz AA, Meldrum C, Curtis JL, Cooper CB, Pirozzi $\mathrm{C}$, et al. Age and small airway imaging abnormalities in subjects with and without air flow obstruction in SPIROMICS. Am J Respir Crit Care Med. 2017;195:464-72. doi: 10.1164/ rccm.201604-08710C.

8. Winter DH, Manzini M, Salge JM, Busse A, Jaluul O, Jacob Filho W, et al. Aging of the lungs in asymptomatic lifelong nonsmokers: findings on HRCT. Lung. 2015;193:283-90. doi: 10.1007/s00408-015-9700-3.

9. Copley SJ. Morphology of the aging lung on computed tomography. J Thorac Imaging. 2016;31:140-50. doi: 10.1097/ RTI.0000000000000211.

10. Dyer C. The interaction of ageing and lung disease. Chron Respir Dis. 2012;9:63-7. doi: 10.1177/1479972311433766.

11. Lee KW, Chung SY, Yang I, Lee Y, Ko EY. Thoracic imaging correlation of aging and smoking with air trapping at thin-section ct of the lung in asymptomatic subjects. Radiology. 2000;214:831-6.

12. Schroder TH, Storbeck B, Rabe KF, Weber C. The aging lung: clinical and imaging findings and the fringe of physiological state. Rofo. 2015;187:430-9. doi: 10.1055/s-0034-1399227.

13. El-sherief AH, Gilman MD, Healey TT, Tambouret RH, Shepard JO, Abbott GF, et al. Clear vision through the haze: a practical approach to ground-glass opacity. Curr Probl Diagn Radiol. 2014;43:140-58. doi: 10.1067/j.cpradiol.2014.01.004.

14. Henry T, Lin CT, Franks TJ, Galvin JR. Mosaic attenuation: etiology, methods of differentiation, and pitfalls. Radiographics. 2015;35:1360-80. doi: 10.1148/rg.2015140308.
15. Magis-Escurra C, Reijers MH. Bronchiectasis. BMJ Clin Evid. 2015;2015. pii: 1507.

16. Chalmers JD, Aliberti S, Blasi F. Management of bronchiectasis in adults. Eur Respir J. 2015 May;45(5):1446-62. doi: 10.1183/09031936.00119114.

17. Matsuoka S, Uchiyama K, Shima H, Ueno N, Oish S, Nojiri Y. Bronchoarterial ratio and bronchial wall thickness on high-resolution CT in asymptomatic subjects: correlation with age and smoking. AJR Am J Roentgenol. 2003;180:513-8.

18. Mercado N, Ito K, Barnes PJ. Accelerated ageing of the lung in COPD: new concepts. Thorax. 2015;70:482-9. doi: 10.1136/ thoraxjnl-2014-206084.

19. Diaz AA, Young TP, Maselli DJ, Martinez CH, Maclean ES, Yen $\mathrm{A}$, et al. Bronchoarterial ratio in never-smokers adults: Implications for bronchial dilation definition. Respirology. 2017;22:108-13. doi: 10.1111/resp.12875.

20. Kang E, Lee JW, Choo JY, Yong HS, Lee KY, Oh Y. The normal aging chest: an overview of radiologic imaging features. J Korean Soc Radiol.2017;77:166-76. 